\title{
Effet de la progestérone sur le taux d'ovulation et la durée du cycle ovarien induits par effet mâle chez la brebis Barbarine et la chèvre locale tunisienne
}

\author{
N Lassoued 1, G Khaldi 1, Y Cognié 2, \\ $P$ Chemineau 2 , J Thimonier 3
}

1 INRAT, laboratoire de recherches ovines et caprines, 2080 Ariana, Tunisie;
2 INRA, station de physiologie de la reproduction, 37380 Nouzilly;
${ }^{3}$ ENSAM, département de zootechnie, place Viala, 34060 Montpellier, France

(Reçu le 21 juin 1994; accepté le 10 mai 1995)

Résumé - Deux expériences ont été conduites sur 30 brebis Barbarines (exp 1) et sur 40 chèvres locales tunisiennes (exp 2) en anœstrus saisonnier. Dans chaque expérience, $20 \mathrm{mg}$ de progestérone ont été administrés par voie intramusculaire au moment de l'introduction des mâles (JO) chez la moitié des femelles, l'autre moitié constituant le lot témoin. Des échantillons de sang ont été prélevés toutes les $4 \mathrm{~h}$ pendant $72 \mathrm{~h}$ pour déterminer le moment d'apparition du pic préovulatoire de $\mathrm{LH}$ en réponse à l'«effet bélier» (exp 1) et 1 fois par jour entre $\mathrm{J} 1$ et $\mathrm{J} 8$ pour suivre l'évolution de la progestérone plasmatique $(\exp 1, \exp 2)$. Les taux d'ovulation ont été déterminés par endoscopie à $\mathrm{J} 4$ et J9 et les comportements de chaleurs contrôlés 2 fois par jour dans les 2 expériences. Chez la brebis, le traitement à la progestérone a retardé le moment d'apparition du pic préovulatoire de $\mathrm{LH}(58,8 \pm 10,1$ vs $20,5 \pm 10,7$ heures; $P<0,001$ ), et supprimé totalement la formation de corps jaunes hypofonctionnels $(0 / 15$ vs $7 / 14, P<0,001)$. Le taux d'ovulation induit n'est pas significativement différent chez les femelles témoins et traitées (respectivement 1,50 $\pm 0,52$ vs $1,26 \pm 0,46$ ). L'œstrus a accompagné l'ovulation induite chez uniquement une femelle témoin et 2 traitées. La suppression des cycles courts par le traitement progestérone a permi la synchronisation des chaleurs sur une période de $3 \mathrm{j}$ ( $93 \mathrm{vs}$ $40 \%)$ au lieu de 9 dans le lot témoin $(P<0,001)$. Chez la chèvre, toutes les ovulations induites dans le lot témoin ont été suivies d'un cycle ovulatoire de courte durée. Dans le lot traité avec la progestérone, 3 chèvres sur 20 ont développé des corps jaunes induits anormaux $(P<0,001)$. L'apparition des $1^{\text {res }}$ chaleurs a eu lieu entre $\mathrm{J} 1$ et $\mathrm{J} 8 \mathrm{chez}$ les témoins, et entre $\mathrm{J} 2$ et $\mathrm{J} 3 \mathrm{chez}$ les traitées. Le traitement progestérone a augmenté, le pourcentage de femelles montrant un œstrus à l'ovulation induite (100 vs $35 \%, P<0,001)$, et le taux d'ovulation au $1^{\text {er }}$ œstrus $(1,85$ vs $1,53, P<0,001)$. En conclusion, l'injection de progestérone au moment de l'introduction des mâles est une technique efficace pour 
supprimer les cycles ovariens de courte durée induits par effet mâle chez la brebis et la chèvre. En plus chez la chèvre, la progestérone augmente le pourcentage de femelles en cestrus et le taux d'ovulation au cours du premier cycle ovarien induit par l'«effet bouc".

effet mâle / taux d'ovulation / corps jaune / brebis / chèvres / progestérone

Summary - Effect of progesterone on ovulation rate and oestrus cycle length induced by the male effect in the Barbarine ewe and Tunisian local goat. Two experiments were conducted on 30 Barbarine ewes (Exp 1) and 40 local Tunisian goats (Exp 2) in seasonal anoestrus (May). In both experiments, half of the females received $20 \mathrm{mg}$ of progesterone intramuscularly just before male introduction (day 0). Blood samples were taken at $4 \mathrm{~h}$ intervals from the time of introduction of the ram, for the determination of the time of the preovulatory LH surge in response to the 'ram effect' (Exp 1) and once a day from days 1 to 8 to determine plasmatic progesterone concentrations (Exp 1, Exp 2). The ovulation rate was determined by coelioscopy at days 4 and 9 , and œestrus behaviour was monitored twice daily in each experiment. In the ewe, progesterone delayed the time of the preovulatory $L H$ surge $(58.8 \pm 10.1$ vs $20.5 \pm 10.7 \mathrm{~h}, \mathrm{P}<0.001)$, and suppressed hypofunctional corpora lutea $(0 / 15$ vs $7 / 14, P<0.001)$. The induced ovulation rate was not significantly different between control and treated females (1.50 \pm 0.52 vs $1.26 \pm 0.46$ respectively). Only 1 female in the control group and 2 in the treated group showed œestrus behaviour at the induced ovulation. Suppression of short cycles by progesterone treatment allowed synchronization of oestrus between days 17 and 20 compared to days 14 and 23 in the control group $(\mathrm{P}<0.001)$. In the goat, all induced ovulations in the control group were followed by a short cycle. in the progesterone-treated group, 3 out of 20 goats developed abnormal induced corpora lutea $(\mathrm{P}<0.001)$. First oestrus occurred between days 1 and 8 in control group and between days 2 and 3 in treated goats. Treatment with progesterone increased the percentage of females showing oestrus at induced ovulation (100 vs $35 \%, \mathrm{P}<0.001$ ). The ovulation rate at first oestrus was increased in treated goats (1.85 vs $1.35, \mathrm{P}<0.001)$. In conclusion, $20 \mathrm{mg}$ progesterone administered at day 0 is efficient at preventing the occurrence of short ovarian cycles. After ovulation induced by the male effect, the percentage of females in oestrus, and the induced ovulation rate are increased by progesterone treatment.

male effect / ovulation rate / corpus luteum / ewe / goat / progesterone

\section{INTRODUCTION}

L'induction de l'ovulation par effet mâle est particulièrement intéressante dans les pays méditerranéens où la majorité des races ovines ne présentent pas d'anœstrus intense (Khaldi, 1984). La première ovulation n'est pas accompagnée d'œestrus (Schinckel, 1954), et la durée de vie du corps jaune est variable (Oldham et Martin, 1978 ; Lassoued et Khaldi, 1993). Le cycle ovulatoire de courte durée induit par "effet mâle» chez des brebis en anœstrus saisonnier a déjà été décrit par Oldham et Martin (1978) et Khaldi (1984). Ce phénomène est aussi connu chez la chèvre (Chemineau, 1983, 1985) à la suite de l'introduction de boucs dans un troupeau de chèvres préalablement isolées. Une phase lutéale de courte durée est également associée au premier cycle ovarien induit par des injections séquentielles de GnRH (Southee et al, 1988) ou de LH (Oussaid et al, 1993) chez des brebis en anœstrus.

La fréquence des cycles courts induits par effet mâle au sein d'un groupe de femelles en ancestrus dépend de «l'intensité de l'anœstrus" des brebis (Khaldi, 1984) et des chèvres (Chemineau, 1986). Plus l'ancestrus est profond, plus la fréquence des cycles courts est élevée. L'intensité de l'ancestrus peut être définie par la proportion de femelles ovulant spontanément avant l'introduction des mâles (Lindsay et Signo- 
ret, 1980). D'après Oussaïd et al (1993) l'ancestrus léger est caractérisé par des niveaux plasmatiques relativement élevés de FSH et la présence de follicules normaux capables d'ovuler en réponse à des injections répétées de $\mathrm{LH}$. La fréquence des cycles courts induits par effet mâle peut être augmentée en diminuant les apports nutritionnels au cours de la période d'anœstrus (Khaldi, 1984 ; Lassoued et Khaldi, 1993).

L'origine de l'hypofonctionnalité d'un certain nombre de corps jaunes induits par effet mâle n'est pas encore entièrement élucidée. Elle peut dépendre du degré de maturité des follicules au moment de l'introduction des mâles (Cognié et al, 1982 ; Pearce et al, 1985) ou de la sécrétion prématurée par l'utérus de la prostaglandine F $2 \alpha$, dont l'action lutéolytique est bien démontrée (Goding, 1974). L'ocytocine sécrétée par le corps jaune stimule les sécrétions de PGF2 $\alpha$ par l'utérus (McCraken, 1980). La majorité des pulses de PGF2a coincident avec des élévations de la concentration plasmatique d'ocytocine (Flint et Sheldrick, 1983) et du nombre des récepteurs à l'ocytocine dans l'endomètre au moment de la lutéolyse (Sheldrick et Flint, 1983). La progestérone et les œstrogènes jouent un rôle important dans le déclenchement du mécanisme de la lutéolyse par l'intermédiaire des récepteurs à l'ocytocine présents dans l'endomètre. Un prétraitement à la progestérone diminue la concentration de ces récepteurs et affecte la réponse de PGF2 $\alpha$ à l'ocytocine (Vallet et al, 1990).

Ces cycles courts ne sont pas observés chez les femelles quand l'effet mâle est précédé d'un traitement progestatif sous forme d'éponges vaginales imprégnées d'acétate de fluorogestone chez la brebis (Oldham et al, 1980 ; Cognié et al, 1982) ou chez les chèvres (Chemineau, 1985). Le but du présent travail a été d'examiner les effets d'une injection de $20 \mathrm{mg}$ de progestérone au moment de l'introduction des mâles sur certains paramètres de reproduction chez la brebis de race Barbarine et la chèvre de race locale.

\section{MATÉRIEL ET MÉTHODES}

\section{Détermination de l'état ovarien avant l'introduction des mâles}

Un jour avant l'introduction des mâles $(J-1)$, une endoscopie, réalisée sur les brebis $(n=96)$ et les chèvres $(n=40)$, a permis de séparer les femelles non cycliques des femelles spontanément cycliques.

Chez la brebis, le pourcentage de femelles spontanément cycliques au printemps a été diminué en contrôlant les niveaux alimentaires et l'évolution du poids vif avant et après la mise bas d'automne (Lassoued et Khaldi, 1993).

Plus de la moitié des brebis examinées avaient ovulé avant l'introduction des mâles (51/96). En revanche, aucune chèvre ne présentait de corps jaune à $\mathbf{J}-1$.

\section{Animaux}

\section{Expérience 1 : brebis Barbarines}

L'expérience a été réalisée sur 30 brebis non cycliques de race Barbarine (à grosse queue) âgées de 3 à 6 ans, ayant un poids moyen de $42 \mathrm{~kg}$.

\section{Expérience 2 : chèvres locales}

L'expérience a été réalisée sur 40 chèvres adultes appartenant à la race locale tunisienne et pesant en moyenne $38 \mathrm{~kg}$.

\section{Mise en lots, traitements}

Pour chacune des 2 espèces, les femelles ont été réparties également en 2 groupes ( $n=15$ brebis et 20 chèvres). Ces femelles ont été soumises à l'effet mâle (lot témoin ; I) ou ont reçu en plus une injection intramusculaire de $20 \mathrm{mg}$ de pro- 
gestérone diluée dans de l'huile neutralisée, immédiatement avant l'introduction des mâles (lot traité ; II).

Les mâles, munis d'un tablier, ont été introduits au jour zéro (J0) dans les 2 lots à raison de 1 pour 10 femelles. De J0 à J24 (exp 1) et de J0 à J30 (exp 2), la détection des œstrus était assurée 2 fois par jour. L'immobilisation des femelles au moment du chevauchement par le mâle a été considérée comme critère indicatif de l'oestrus.

L'observation directe des ovaires par endoscopie a permis de dénombrer les corps jaunes, de relever leur taille et leur couleur à $\mathrm{J} 4$ et $\mathrm{J} 9$. Une endoscopie a été effectuée à J14 pour le lot traité à la progestérone car ce traitement retarde les ovulations induites par rapport à celles du lot témoin (Cognié et al, 1982). Huit à 12 jours après l'apparition des chaleurs, une endoscopie a été effectuée pour déterminer le taux des ovulations accompagnées d'œstrus. Le moment d'ovulation et l'âge des corps jaunes sont déterminés d'après leur taille et leur couleur, selon les critères décrits par Oldham et Lindsay (1980). La durée du cycle a été définie par l'intervalle en jours entre 2 ovulations successives.

\section{Prélèvements et dosages}

Dans les 2 expériences le sang a été prélevé à la veine jugulaire, centrifugé, et le plasma a été congelé à $-20^{\circ} \mathrm{C}$ jusqu'aux analyses.

Pour évaluer la durée de vie du corps jaune, le niveau de sécrétion de progestérone a été déterminé dans des prélèvements effectués 1 fois par jour de J1 à J8 (exp 1) et de J1 à J12 (exp 2).

Dans le but de situer le moment d'apparition du pic préovulatoire de $\mathrm{LH}$, des prélèvements ont été effectués toutes les $4 \mathrm{~h}$, pendant $72 \mathrm{~h}$ à partir de J0 (exp 1). Le moment d'apparition du pic préovulatoire a été défini comme étant le premier prélèvement où la concentration de LH s'élève et se maintient à un niveau supérieur à $10 \mathrm{ng} / \mathrm{ml}$ en 2 points successifs (Pearce et al, 1987).

Le progestérone a été dosée avec les kits fournis par l'Agence internationale de l'énergie atomique. La sensibilité du dosage est de 0,09 $\mathrm{ng} / \mathrm{ml}$. Le coefficient de variation intra-dosage était de $10 \%$ et celui inter-dosages de $11,6 \%$.

La LH a été dosée selon la méthode de Pelletier et al (1982), modifiée par Mongomery et al
(1985). La sensibilité du dosage est de $0,1 \mathrm{ng} / \mathrm{ml}$, le coefficient de variation intra-dosage était de $9,2 \%$ et celui inter-dosages de $11,4 \%$.

\section{Analyse statistique}

La comparaison des résultats entre les lots a été effectuée à l'aide du logiciel SAS en utilisant l'analyse de variance pour les concentrations hormonales (proc GLM), et le test $\chi^{2}$ pour les taux d'ovulation et les distributions des chaleurs. Les résultats sont exprimés en termes de moyenne \pm écart type.

\section{RÉSULTATS}

\section{Expérience 1 : brebis Barbarine}

\section{Intensité de l'ancestrus saisonnier, taux d'ovulation et durée du cycle induit}

Sur les 30 brebis en ancestrus soumises à l'effet mâle, 29 ont ovulé après l'introduction des béliers. La seule brebis n'ayant pas ovulé appartient au lot témoin. Dans le lot témoin, la moitié des brebis ont présenté un corps jaune régressant prématurément (tableau I). La durée moyenne du premier cycle ovarien a été de $4,7 \pm 1,2$ j pour les cycles courts, et de 15,0 $\pm 1,0 \mathrm{j}$ pour les cycles normaux. Après le traitement à la progestérone, les cycles ont eu une durée moyenne de $16,9 \pm 0,9$ jours. La proportion de cycles normaux est plus élevée chez les femelles traitées à la progestérone $(P<$ 0,001 ), mais leur durée n'est pas significativement différente de celle des femelles du groupe témoin $(P>0,05)$.

\section{Pic préovulatoire de LH (fig 1)}

Le moment moyen d'apparition du pic préovulatoire de LH est de $20,5 \pm 10,7 \mathrm{~h}$ après l'introduction des béliers dans le lot témoin, 
Tableau I. Nombre de brebis ayant ovulé, taux d'ovulation moyen \pm sd, et pourcentage de cycles courts induits après l'introduction des béliers chez la brebis Barbarine en anœstrus saisonnier, traitée (lot II) ou non à la progestérone (lot I).

\begin{tabular}{|c|c|c|c|c|c|}
\hline \multirow[t]{2}{*}{ Lot } & \multirow[t]{2}{*}{$N^{*}$} & \multirow{2}{*}{$\begin{array}{c}\text { Nombre de brebis } \\
\text { ovulant }\end{array}$} & \multirow{2}{*}{$\begin{array}{c}\text { N cycles courts } \\
(\%)\end{array}$} & \multicolumn{2}{|c|}{ Taux d'ovulation } \\
\hline & & & & Induit & Après fer œestrus \\
\hline $1^{* \star}$ & 15 & 14 & $7(50)^{a}$ & $\begin{array}{c}1,50 \pm 0,52 \\
(14)\end{array}$ & $\begin{array}{l}1,29 \pm 0,47 \\
(14)\end{array}$ \\
\hline II *** & 15 & 15 & $0(50)^{b}$ & $\begin{array}{c}1,26 \pm 0,46 \\
(15)\end{array}$ & $\begin{array}{l}1,26 \pm 0,46 \\
(15)\end{array}$ \\
\hline
\end{tabular}

* Nombre de brebis soumises à l'effet mâle ; ${ }^{\star \star}$ lot témoin ; ${ }^{\star \star \star}$ lot ayant reçu $20 \mathrm{mg}$ de progestérone; ${ }^{\mathrm{a}}$ vs ${ }^{\mathrm{b}} P<0,001$.

et $58,9 \pm 10,1 \mathrm{~h}$ dans le lot traité à la progestérone $(P<0,001)$. Le pic préovulatoire apparaît décalé de près de 2 j chez les femelles traitées à la progestérone.

Dans le lot témoin, les femelles ayant un cycle induit de courte durée ont présenté un moment moyen d'apparition du pic de LH qui n'est pas significativement différent de celui des femelles chez lesquelles un cycle normal a été observé (respectivement $17,1 \pm 8,2$ vs $23,5 \pm 12,6$ h).
Les taux d'ovulation induits ont été de $1,50 \pm 0,52$ et $1,26 \pm 0,46$ et ceux après le premier œstrus de $1,29 \pm 0,47$ et $1,26 \pm$ 0,46 , respectivement dans les lots témoin et traité (tableau I). Aucune de ces différences n'est significative au seuil de $5 \%$.

\section{Apparition du comportement de chaleurs}

La répartition des chaleurs au cours des 23 j qui ont suivi l'introduction des béliers a
Fig 1. Évolution de la concentration plasmatique moyenne de $\mathrm{LH}$ en $\mathrm{ng} / \mathrm{ml}$ après introduction des béliers chez les brebis témoins et traitées à la progestérone.
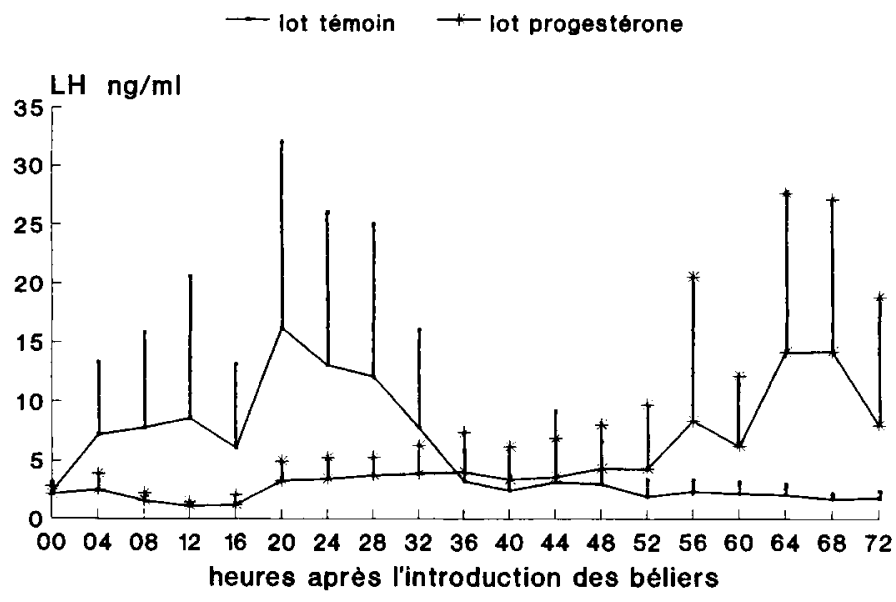
été différente dans les 2 lots $(P<0,001$; fig 2). En effet, dans le lot traité à la progestérone, la première ovulation induite a été accompagnée de chaleurs chez 2 brebis (soit 13\%). Les chaleurs ont été groupées entre le $17^{\mathrm{e}}$ et le $20^{\mathrm{e}}$ jour après l'introduction des mâles, avec un maximum de fréquence au 19 e jour. En revanche, dans le lot témoin, l'ovulation induite a été accompagnée de chaleurs chez une seule brebis (soit $7 \%$ ). Les chaleurs étant moins synchronisées, 2 groupes de brebis peuvent être distingués. En effet, les chaleurs sont apparues entre le $14^{e}$ et le $16^{e}$ jour pour 6 brebis (soit $43,8 \%$ ) et entre le $20^{\circ}$ et le $23^{e}$ jour pour 7 brebis (soit 50\%).

\section{Évolution de la progestérone plasmatique}

L'évolution de la concentration de progestérone plasmatique est représentée à la figure 3 . À J4, chez les brebis témoins, cette concentration n'est pas différente pour les brebis ayant un corps jaune induit normal ou de courte durée de vie $(0,4 \mathrm{ng} / \mathrm{ml})$. Dans ce groupe, la concentration augmente jusqu'à $2,0 \mathrm{ng} / \mathrm{ml}$ à $\mathrm{J} 8$ quand le cycle induit est de durée normale, et n'est que de $0,1 \mathrm{ng} / \mathrm{ml}$ chez celles qui ont un cycle court $(P<$ $0,001)$. Dans le lot traité, à J1, la progestérone plasmatique est élevée $(1,2 \mathrm{ng} / \mathrm{ml})$ à cause de la progestérone exogène injectée à J0. Le retour au niveau de base est observé à J3. Dans le lot traité à la progestérone, le niveau de $0,4 \mathrm{ng} / \mathrm{ml}$ n'est atteint que $6 \mathrm{j}$ après l'introduction des mâles.

\section{Expérience 2 : chèvre locale}

Intensité de l'ancestrus, taux d'ovulation et durée du cycle induit

Toutes les chèvres des 2 lots ont répondu à l'effet bouc. Le taux d'ovulation induit a été de $1,45 \pm 0,51$ dans le lot témoin et de $1,82 \pm 0,39$ dans le lot traité $(P<0,01)$.

Cette première ovulation induite a été suivie d'un cycle court chez $100 \%$ des chèvres du lot témoin et seulement $15 \%$ des chèvres traitées à la progestérone (tableau II ; $P<0,001$ ). La durée moyenne des cycles courts a été de $5,6 \pm 1,2$ et $6,0 \pm$ $0,0 \mathrm{j}$ respectivement dans les lots témoin et traité. La durée moyenne des 17 cycles normaux dans le lot traité a été de 21,0 $0,9 \mathrm{j}$.

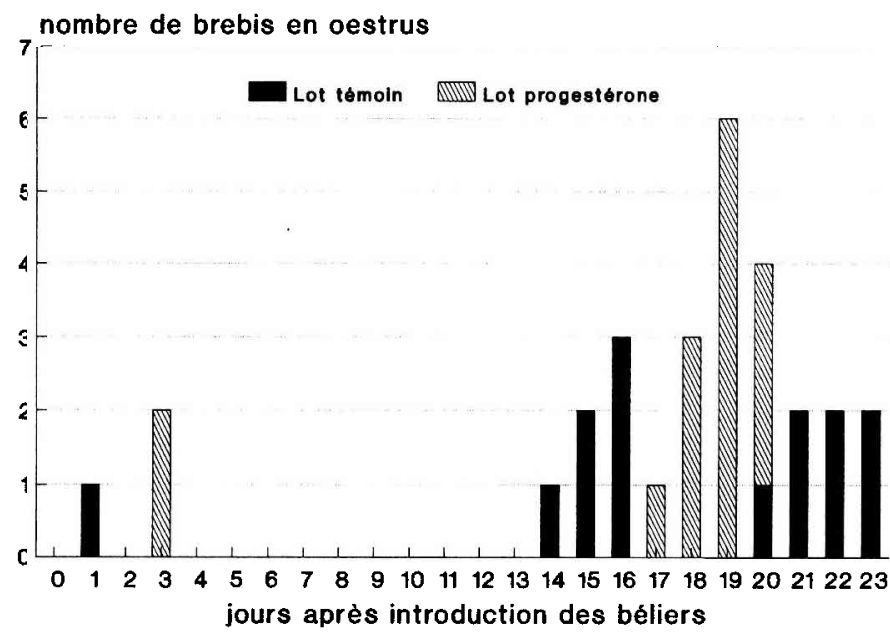

Fig 2. Distribution des chaleurs après introduction des béliers chez les brebis témoins et traitées à la progestérone. 


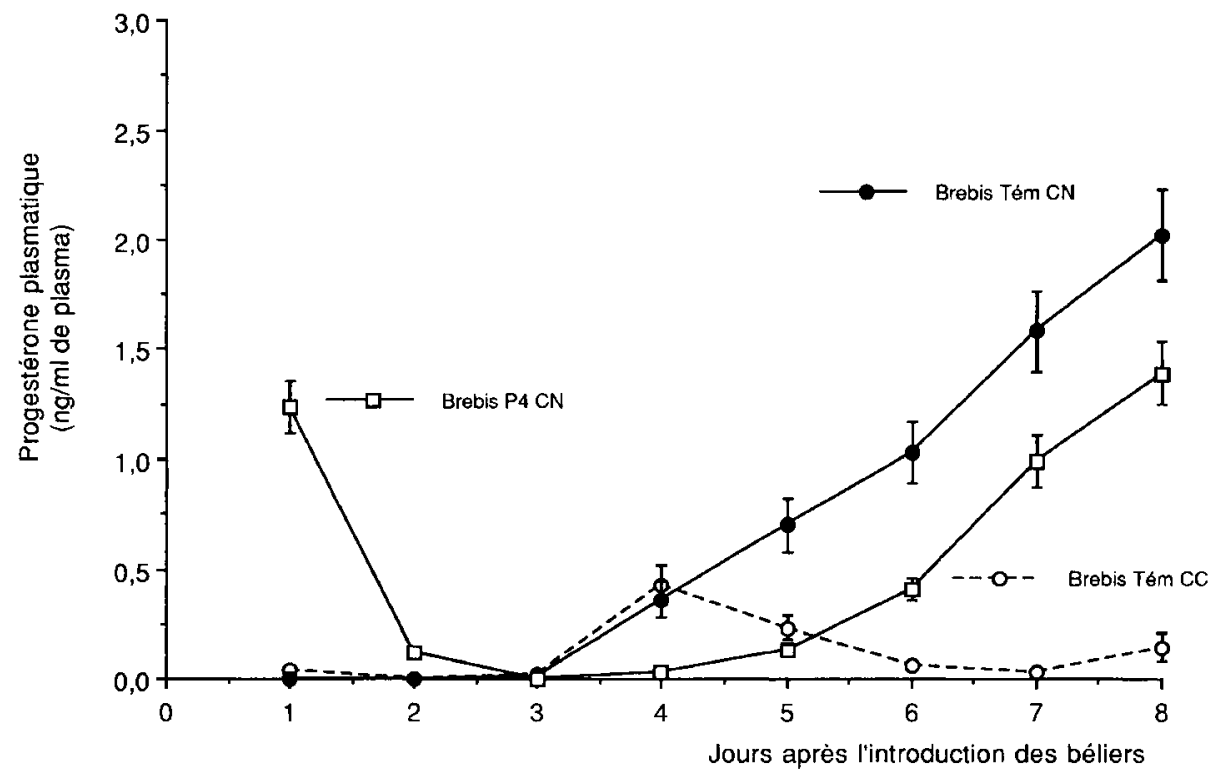

Fig 3. Évolution de la progestérone plasmatique en $(\mathrm{ng} / \mathrm{ml})$ après introduction des béliers, chez les brebis des lots témoin et traité à la progestérone.

\section{Apparition du comportement de chaleurs}

La répartition des chaleurs après l'introduction des boucs est indiquée à la figure 4 et est différente selon les groupes de trai- tement $(P<0,001)$. La première ovulation induite est accompagnée d'œstrus chez seulement 7 femelles du lot témoin (soit $35 \%)$; en revanche, dans le lot traité à la progestérone, toutes les femelles manifes-

Tableau II. Nombre de chèvres ayant ovulé, taux d'ovulation moyen \pm sd, et pourcentage de cycles courts induits après l'introduction des boucs chez la chèvre locale tunisienne traitée (lot II) ou non traitée à la progestérone (lot I).

\begin{tabular}{|c|c|c|c|c|c|c|}
\hline \multirow[t]{2}{*}{ Lot } & \multirow{2}{*}{$\begin{array}{l}\text { Nombre de } \\
\text { chèvres ovulant }\end{array}$} & \multirow{2}{*}{$\begin{array}{l}\mathrm{N} \text { cycles } \\
\text { courts (\%) }\end{array}$} & \multicolumn{2}{|c|}{ Femelles en œstrus } & \multicolumn{2}{|c|}{ Taux d'ovulation } \\
\hline & & & fre ovulation & $2^{e}$ ovulation & Induit & Après 1er cestrus \\
\hline $1 *$ & 20 & $20(100)^{a}$ & 7 & 19 & $\begin{array}{c}1,45 \pm 0,51^{c} \\
(20)\end{array}$ & $\begin{array}{c}1,53 \pm 0,51^{c} \\
(19)\end{array}$ \\
\hline $11 * *$ & 20 & $3(15)^{b}$ & 20 & 17 & $\begin{array}{c}1,85 \pm 0,36 d \\
(20)\end{array}$ & $\begin{array}{c}1,85 \pm 0,36 \mathrm{~d} \\
(20)\end{array}$ \\
\hline
\end{tabular}

* Lot témoin ; ${ }^{* *}$ lot ayant reçu $20 \mathrm{mg}$ progestérone ; ${ }^{\mathrm{c}} v \mathrm{~s}^{\mathrm{d}} P<0,01$; ${ }^{\mathrm{a}}$ vs ${ }^{\mathrm{b}} P<0,001$. 


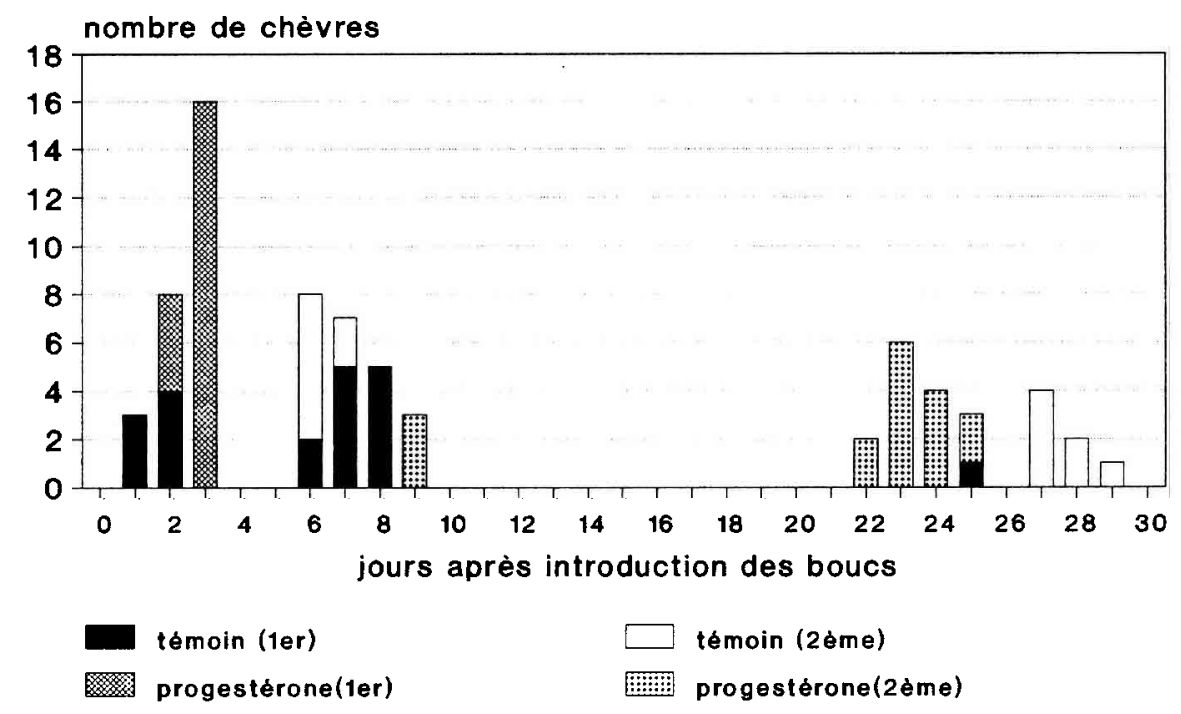

(1er) : ler oestrus

(2ème): 2ème oestrus

Fig 4. Distribution des 1 ers et $2^{\text {nds }}$ œstrus chez les chèvres témoins et traitées à la progestérone (P4) après introduction des boucs.

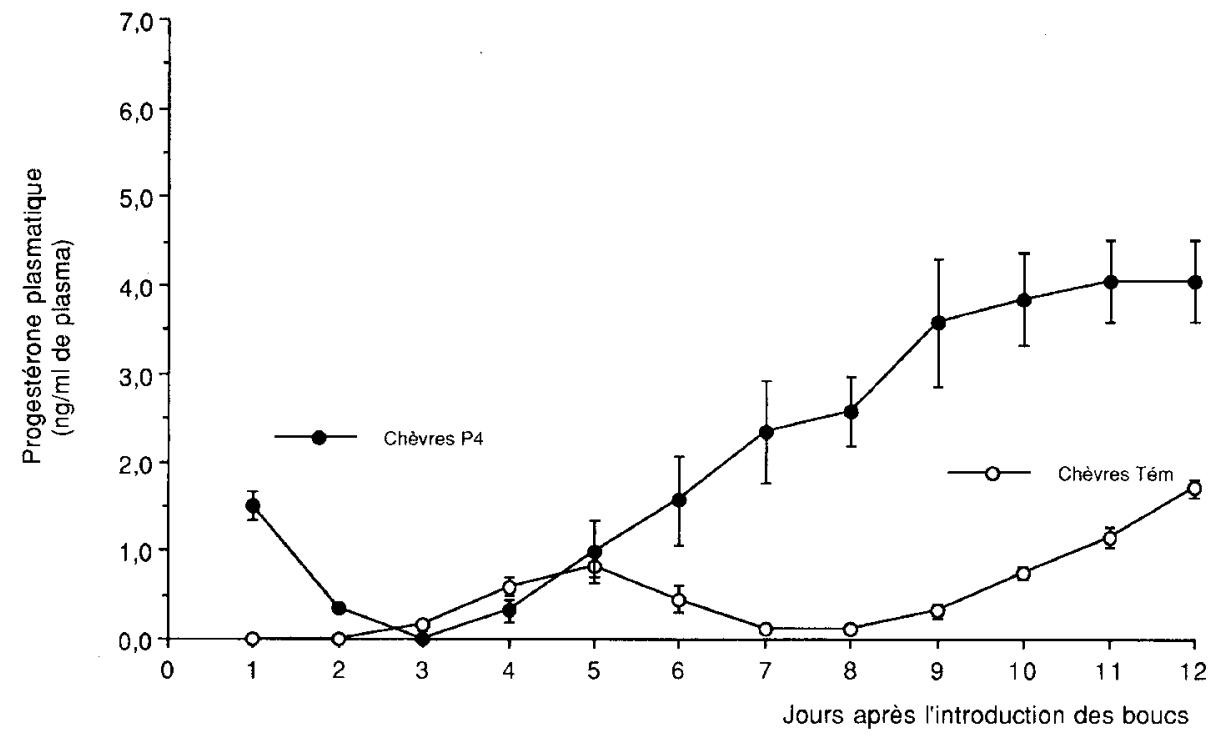

Fig 5. Évolution de la concentration de la progestérone plasmatique (en $\mathrm{ng} / \mathrm{ml}$ ) après introduction des boucs, chez les chèvres des lots témoin et traité à la progestérone. 
tent un comportement de chaleur accompagnant l'ovulation induite. Dans le lot témoin, les premiers cestrus sont répartis entre J1 et J8. En revanche dans le lot traité, ils se manifestent entre $\mathrm{J} 2$ et $\mathrm{J} 3$ avec un maximum à $\mathrm{J} 3(n=16 / 20)$. L'apparition des deuxièmes chaleurs est également différente dans les 2 lots $(P<0,001)$. En effet, ces retours sont observés au cours de 2 périodes, de J6 à $\mathrm{J} 7$ et de J27 à J29 après l'introduction des boucs chez les femelles n'ayant reçu aucun traitement. Ce second œstrus est plus synchronisé (entre J22 et J25) chez les femelles ayant reçu la progestérone que chez les femelles témoins. Les 3 femelles en œstrus à J9 sont celles ayant présenté un cycle de courte durée. Trois autres chèvres du même lot n'ont pas manifesté de retour en œstrus.

\section{Concentration de la progestérone plasmatique}

Les cycles courts observés chez les femelles témoins sont accompagnés d'une élévation de progestérone plasmatique à partir de $\mathrm{J} 3$ avec un maximum de $0,8 \mathrm{ng} / \mathrm{ml}$ à J5. Cette élévation est transitoire, puisque la concentration est redescendue au niveau de base à J8 (fig 5). Ce cycle court est toujours suivi d'un deuxième, de durée normale. Dans le lot traité à la progestérone, l'activité des corps jaunes a été normale dans $85 \%$ des cas, mais l'élévation du niveau de progestérone a commencé à $\mathrm{J} 4$, soit $1 \mathrm{j}$ plus tard que dans le groupe témoin.

\section{DISCUSSION}

La race Barbarine présente au printemps un anœstrus saisonnier peu intense (Khaldi, 1984). En effet, 25 à $40 \%$ des brebis continuent à avoir une activité ovarienne et sont observés en œstrus. Ce taux d'anœstrus peut présenter des variations très impor- tantes d'une année à l'autre. En effet, ce taux peut varier de $0 \%$ en année pluvieuse à $100 \%$ en année très sèche (Lassoued et Khaldi, résultats non publiés). En revanche, dans l'espèce caprine, l'absence de l'activité ovarienne et de manifestations comportementales est quasi totale pendant la même période et ne semble pas être soumise à des variations annuelles.

L'effet mâle est une technique utilisée pour rétablir l'activité ovarienne et la comportement d'œstrus et permettre d'obtenir des mises bas aux moments favorables de l'année. Des travaux antérieurs ont montré que la réponse à l'effet mâle dépend de l'intensité de l'anœstrus qui peut être appréciée par le pourcentage de femelles ovulant spontanément avant l'introduction du mâle (Lindsay et Signoret, 1980).

L'ovulation induite par l'introduction des mâles est suivie par une phase lutéale de courte durée. Le pourcentage de ces cycles courts est fortement influencé par le niveau alimentaire des brebis et par l'intensité de I'anoestrus avant l'introduction des mâles (Khaldi, 1984; Lassoued et Khaldi, 1993). En revanche chez les chèvres, ce pourcentage d'apparition des cycles courts est plus stable. Toutes les chèvres soumises à l'effet mâle ont un cycle induit de courte durée.

Ces différences entre espèces ou interannuelles chez la brebis pourraient être dues à une inhibition plus ou moins forte de l'axe hypothalamus-hypophyse ovaire. Oussaïd et al (1993) ont montré qu'il existe une différence de réponse de l'ovaire stimulé par un traitement séquentiel de $\mathrm{LH}$ pendant l'ancestrus léger ou profond. L'ancestrus léger se caractérise par un taux plus élevé de FSH et la présence d'une plus grande proportion de follicules non atrétiques capables d'ovuler après stimulation par LH.

Ces cycles anormalement courts ne sont pas observés quand l'effet mâle est associé à un traitement aux éponges vaginales 
imprégnées d'acétate de fluorogestone (brebis : Oldham et al, 1980, 1985 ; Lindsay et al, 1982 ; Cognié et al, 1982 ; chèvre : Chemineau, 1985).

Les résultats de nos expériences sur des races tunisiennes montrent qu'une injection de $20 \mathrm{mg}$ de progestérone immédiatement avant l'introduction des mâles dans un troupeau de femelles en ancestrus saisonnier n'a pas d'effet négatif sur leurs taux de réponse. En outre, elle permet de prévenir l'apparition des cycles courts, ce qui est en accord avec les résultats publiés par d'autres auteurs sur des races ovines (Cognié et al, 1982 ; Oldham et al, 1985) ou caprines (Chemineau, 1985).

L'existence des cycles induits de courte durée est à l'origine de la dispersion des comportements de chaleurs observée dans les lots témoins chez les 2 espèces. L'injection de progestérone permet un groupage des œstrus entre J17 et J20 chez la brebis, et entre $\mathrm{J} 2$ et $\mathrm{J} 3 \mathrm{chez}$ la chèvre. II est important de signaler que le traitement progestatif augmente le pourcentage de chèvres en œstrus à l'ovulation induite par effet bouc. Ce phénomène a été signalé chez la chèvre créole avec I'utilisation d'éponges imprégnées de FGA, ou l'injection de $5,2 \mathrm{mg}$ de FGA immédiatement avant l'introduction des boucs (Chemineau, 1985).

Par ailleurs, la progestérone améliore significativement le taux d'ovulation chez la chèvre et pas chez la brebis. D'autres auteurs ont déjà mentionné cette absence d'effet chez la brebis (Lindsay et al, 1982). On peut se demander si ces différences entre chèvres et brebis (sur l'augmentation du pourcentage de femelles en cestrus et du taux d'ovulation induit après injection de progestérone) sont liées à une différence entre espèces des mécanismes aboutissant à la sélection des follicules préovulatoires.

En effet, le pic préovulatoire de LH est retardé de $38 \mathrm{~h}$ environ chez les brebis traitées à la progestérone, ce qui permet d'al- longer le temps de maturation des follicules préovulatoires sous l'effet des hormones gonadotropes (Martin et al, 1983 ; Pearce et al, 1985).

Chez la chèvre, le moment d'apparition du pic préovulatoire de LH n'a pas été précisé dans cette expérience. En revanche, le pic des premiers œstrus accompagnant l'ovulation induite est retardé de $1 \mathrm{j}$ dans le lot traité à la progestérone (J3) par rapport aux chèvres témoins $(\mathrm{J} 2)$. Ce résultat nous laisse supposer que l'ovulation induite est également retardée de $1 \mathrm{j}$.

L'hypothèse selon laquelle l'augmentation de l'intervalle effet mâle-ovulation est responsable de la suppression des cycles courts a été l'objet de nombreuses discussions. Les travaux de Martin et al (1981) ont montré que, même si l'injection est faite 2 j avant l'introduction des béliers, ce qui ne provoque aucun retard dans le déclenchement du pic préovulatoire de $\mathrm{LH}$, les cycles courts sont également supprimés. La progestérone pourrait donc agir directement sur le développement des follicules préovulatoires. Brown et al (1988) ont montré chez des brebis en ancestrus une corrélation positive entre le flux sanguin au niveau des capillaires ovariens et la fonction lutéale induite. Ce flux est significativement augmenté pendant la phase préovulatoire par l'injection de $20 \mathrm{mg}$ de progestérone.

L'action de la progestérone n'est probablement pas limitée au niveau ovarien. La prostaglandine $\mathrm{F} 2 \alpha$, sécrétée par l'utérus des ruminants, est reconnue depuis longtemps comme le facteur lutéolytique (Goding, 1974). Plusieurs travaux ont mis en évidence le rôle que joue l'utérus dans la régression prématurée des corps jaunes induits chez la brebis en ancestrus (Southee et al, 1988; Hunter et al, 1989). L'injection d'ocytocine provoque une régression du corps jaune en stimulant la sécrétion de PGF2 $\alpha$ (Cooke et Homeida, 1982) et cette sécrétion des PGF2 $\alpha$ est corrélée à 
la concentration des récepteurs à l'ocytocine dans l'endomètre (Mitchell et al, 1975 ; Flint et al, 1990).

Le rôle joué par la progestérone est suggéré par le fait que les récepteurs à l'ocytocine se trouvent en faible quantité pendant la phase lutéale du cycle et augmentent à l'œstrus (Sheldrick et Flint, 1985). En outre, un traitement à la progestérone est suivi d'une baisse de la concentration de ces récepteurs (Vallet et al, 1990 ; Lau et al, 1992a, b). Ce stéroïde aurait une action inhibitrice sur la mise en place des récepteurs à l'ocytocine et donc sur la libération de PGF2 $\alpha$. La présence (brebis non traitées à la progestérone) ou l'absence (brebis traitées) de ces récepteurs à l'ocytocine dans l'endomètre pendant le début de la phase lutéale peut donc déterminer la durée du cycle chez des brebis en anœstrus dont l'ovulation est induite pendant l'anœstrus. Chez les femelles non traitées à la progestérone, la variabilité dans la durée du cycle induit pourrait être à la fois liée à la maturation folliculaire et au degré d'inhibition des mécanismes lutéolytiques au niveau de l'utérus.

En conclusion, les résultats de ces expériences sur des races ovines ou caprines tunisiennes montrent que l'utilisation d'un traitement progestatif préalable pour améliorer la réponse à l'effet mâle en supprimant les ovulations de mauvaise qualité permet de grouper les chaleurs et faciliter la pratique de l'insémination artificielle.

\section{REMERCIEMENTS}

Ce travail a été réalisé avec la participation financière de la Coopération technique française et de l'Agence internationale de l'énergie atomique. Les auteurs tiennent à remercier D André (laboratoire des dosages à l'INRA de Nouzilly) pour la réalisation des dosages de la $\mathrm{LH}$, et $\mathrm{A}$ Latifa (INAT, Tunisie) pour l'analyse statistique des résultats.

\section{RÉFÉRENCES}

Brown BW, Cognié $Y$, Chemineau $P$, Poulin N, Salama OA (1988) Ovarian capillary blood flow in seasonally anoestrous ewes induced to ovulate by treatment with GnRH. J Reprod Fert 84, 653-658

Chemineau $P$ (1983) Effect on oestrus and ovulation of exposing creole goats to the male at 3 times of the year. J Reprod Fert 67, 65-72

Chemineau $P$ (1985) Effects of a progestagen on buckinduced short ovarian cycles in the Creole meat goat. Anim Reprod Sci 9, 87-94

Chemineau P (1986) Influence de la saison sur l'activité sexuelle du cabri créole mâle et femelle. Thèse de doctorat USTL, Montpellier

Cognié Y, Gray SJ, Lindsay DR, Oldham CM, Pearce DT, Signoret JP (1982) A new approach to controlled breeding in sheep using the "ram effect". Proc Aust Soc Anim Prod 14, 519-522

Cooke RG, Homeida AM (1982) Plasma concentrations of 13,14 dihydro-15-Keto prostaglandin $\mathrm{F} 2 \alpha$ and progesterone during oxytocin-induced oestrus in the goat. Theriogenology 18, 453-460

Flint APF, Sheidrick EL (1983) Evidence for a systemic role for oxytocin in luteal regression in sheep. $J$ Reprod Fert 67, 215-225

Flint APF, Sheldrick EL, McCann TJ, Jones DSC (1990) Luteal oxytocin: characteristics and control of synchronous episodes of oxytocin and PGF2 $\alpha$ secretion at luteolysis in ruminants. Dom Anim Endocrinol 7, 111-124

Goding JR (1974) The demonstration that PGF2 $\alpha$ is the uterine luteolysin in the ewe. J Reprod Fert 38,261 271

Hunter MG, Ayad VJ, Gilbert CL, Southee JA, Wathes $C D$ (1989) Role of prostaglandin $F 2 \alpha$ and ocytocin in the regression of $\mathrm{GnRH}$-induced abnormal corpora lutea in anoestrus ewes. J Reprod Fert 85 , $551-556$

Khaldi G (1984) Variations saisonnières de l'activité ovarienne du comportement d'œstrus et de la durée de l'ancestrus post-partum des femelles ovines de race Barbarine : influence du niveau alimentaire et de la présence du mâle. Thèse doct ès sciences, USTL, Montpellier

Lassoued N, Khaldi G (1993) Études sur l'influence du niveau alimentaire avant et après la mise bas sur la réponse des brebis de race Barbarine à l'ceffet mâle" en Tunisie. Improving the productivity of indigenous African livestock. Results of FAO/IAEA/DGIS Coordinated Research Programmes organized by the joint FAO/IAEA division of Nuclear Techniques in Food and Agriculture, 59-66

Lau TM, Gow CB, Fairclough RJ (1992a) Increases in the oxytocin-induced prostaglandin $\mathrm{F} 2 \alpha$ response and reduction in the concentrations of endometrial oxy- 
tocin receptors in ewes in response to progesterone $\checkmark$ Reprod Fert 95, 11-18

Lau TM, Kerton DJ, Gow CB, Fairclough RJ (1992b) Increase in concentration of uterine oxytocin receptors and decrease in response to 13,14-dihydro-15keto prostaglandin $F 2 \alpha$ in ewes after withdrawal of exogenous progesterone. J Reprod Fertil 95, 885893

Lindsay DR, Signoret JP (1980) Influence of behaviour on reproduction. 9th Int Cong Anim Reprod Artif Insem, 1, 83-92

Lindsay DR, Cognié Y, Signoret JP (1982) Méthode simplifiée de maîtrise de l'œestrus chez la brebis. Ann Zootech 31, 77-82

Martin GB, Scaramuzzi RJ, Lindsay DR (1981) Induction of ovulation in seasonally anovular ewes by the introduction of rams: effects of progesterone and active immunization against androstenedione. Aust $J$ Biol Sci 34, 569-575

Martin GB, Scaramuzzi RJ, Oldham CM, Lindsay DR (1983) Effects of progesterone on the responses of Merino ewes to the introduction of rams during anoestrus. Aust J Biol Sci 36, 369-378

McCracken JA (1980) Hormone receptor control of PGF2 $\alpha$ secretion by the ovine uterus. Adv Prosta Tromb Res 8, 1329-1344

Mitchell MD, Flint APF, Turnbull AC (1975) Stimulation of oxytocin by protaglandin $F$ levels in uterine venous efluent in pregnant and puerperal sheep. Prostaglandins 9, 47-56

Mongomery GW, Martin GB, Pelletier J (1985) Change in pulsatile $\mathrm{LH}$ secretion after ovariectomy in île-deFrance ewes in 2 seasons. J Reprod Fertil 73, 173183

Oldham CM, Martin GB (1978) Stimulation of seasonally anovular Merino ewes by rams. II. Premature regression of ram-induced corpora lutea. Anim Reprod Sci 1, 283-290

Oldham CM, Lindsay DR (1980) Laparoscopy in the ewe: a photographic record of the ovarian activity of ewes experiencing normal or abnormal oestrus cycles. Anim Reprod Sci 3, 119-124
Oldham CM, Cognié $Y$, Poindron $P$, Gayerie $P$, Gayerie $F(1980)$ The influence of progesterone of $F G A$ priming on the ovarian function of seasonally ewes induced to ovulate by their re-introduction to rams. Proc IXth Int Cong Anim Reprod and Al, 16-20 June, Madrid 3, 50

Oldham CM, Pearce DT, Gray SJ (1985) Progesterone priming and age of ewe affect the life-span of corpora lutea induced in the seasonally anovulatory Merino ewe by the ram effect. J Reprod Fert 75, 29-33

Oussaid B, Cognié Y, Mariana JC (1993) Ovarian stimulation following repeated injections of $\mathrm{LH}_{\text {or }} \mathrm{LH}^{+}$ FSH in île-de-France sheep in early and mid-seasonal anoestrus. Anim Reprod Sci 31, 83-98

Pearce DT, Martin GB, Oldham CM (1985) Corpora lutea with a short life-span induced by rams in seasonally anovulatory ewes are prevented by progesterone delaying the preovulatory surge of $\mathrm{LH}$. J Reprod Fertil 75, 79-84

Pearce DT, Oldham CM, Haresign W, Gray SJ (1987) Effect of duration and timing of progesterone priming on the incidence of corpora lutea with a normal life-span in Merino ewes induced to ovulate by the introduction of rams. Anim Reprod Sci 13, 81-89

Pelletier J, Garnier DH, de Reviers MM, Terqui M, Ortavant $R$ (1982) Seasonal variation in $\mathrm{LH}$ and testosterone release in rams of 2 breeds. J Reprod Fert 64, 341-346

Scheldrick EL, Flint APF (1985) Endocrine control of uterine oxytocin receptors in the ewe. J Endocr 106 , 249-258

Schinckel PG (1954) The effect of the presence of the ram on the ovarian activity of the ewe. Aust J Agric Res 5, 465-469

Southee JA, Hunter MG, Haresing W (1988) Function of abnormal corpora lutea in vivo after $\mathrm{GnRH}$-induced ovulation in the anoestrous ewes. J Reprod Fert 84 , 131-137

Vallet JL, Lamming GE, Batten M (1990) Control of endometrial oxytocin receptor and uterine response to oxytocin by progesterone and oestradiol in the ewe. J Reprod Fert 90, 625-634 